\title{
Severe Persistent Eczema in a Recipient of the Gam-COVID-Vac vaccine
}

\author{
Maryam Ameri ${ }^{1}$, Meysam Abolmaali ${ }^{2}$, Sayed Mohammed Jawad Alwedaie ${ }^{3}$, Mohammad Nabavi ${ }^{4}$, \\ Neda Rahimian ${ }^{5}$, Negin Mahmoodi Hamidabad ${ }^{6}$ \\ ${ }^{1}$ Department of Forensic Medicine, School of Medicine, Iran University of Medical Sciences, Tehran, Iran \\ ${ }^{2}$ Shefa Neuroscience Research Center, Khatam Alanbia Hospital, Tehran, Iran \\ ${ }^{3}$ Manama, Bahrain \\ ${ }^{4}$ Department of Allergy \& Clinical Immunology, School of Medicine, Iran University of Medical Science, Tehran, Iran \\ ${ }^{5}$ Department of Internal Medicine, School of Medicine, Iran University of Medical Science, Tehran, Iran \\ IIran University of Medical Sciences, Tehran, Iran
}

\section{Doi: 10.12890/2022_003042 - European Journal of Case Reports in Internal Medicine - ๑ EFIM 2022}

Received: 31/10/2021

Accepted: 05/12/2021

Published: $12 / 01 / 2022$

How to cite this article: Ameri M, Abolmaali M, Alwedaie SMJ, Nabavi M, Rahimian N, Hamidabad NM. Severe persistent eczema in a recipien to fhe GamCOVID-Vac vaccine. EJCRIM 2022;9: doi:10.12890/2022_003042.

Conflicts of Interests: The authors declare there are no competing interests.

This article is licensed under a Commons Attribution Non-Commercial 4.0 License

\section{ABSTRACT}

Since the beginning of the COVID-19 pandemic, efforts have been made to design safe and effective vaccines against SARS-CoV-2. Numerous vaccines have been designed and tested in limited clinical trials in various countries. Among them, the Sputnik $V$ vaccine has shown a relatively safe profile and, to our knowledge, has no associated major side effects. We describe the case of a 40-year-old female healthcare worker who developed severe persistent eczematous lesions on the second day after she received the first dose of the Sputnik vaccine. The eczematous lesions were refractory to an antihistamine and persisted at the 1 month follow-up. Severe persistent eczematous lesions should be viewed as a potential side effect of vaccination with the Sputnik $\mathrm{V}$ vaccine. Moreover, a severe allergic reaction to a COVID-2019 vaccine may indicate the vaccine is ineffective in the recipient.

\section{LEARNING POINTS}

- Vaccination against COVID-19 may be accompanied by rare complications.

- Eczematous lesions can be a side effect of the Sputnik $V$ vaccine.

- A severe allergic reaction to a COVID-19 vaccine may result in decreased vaccine effectiveness in the recipient.

\section{KEYWORDS}

COVID-19, Sputnik V, eczema

\section{INTRODUCTION}

Since the announcement of the COVID-19 pandemic by the World Health Organization ${ }^{[1]}$ in March 2020, more than 289 million confirmed cases of COVID-19 and approximately 3 million deaths have been recorded ${ }^{[2]}$. Efforts have been made to design safe and effective vaccines against severe acute respiratory syndrome coronavirus 2 (SARS-CoV-2) ${ }^{[3]}$. Despite having numerous benefits, vaccines in general are not completely risk free. The most common side-effects are usually minor, but some can cause serious and life-threatening adverse effects that are not clearly identified until the vaccine is in common use ${ }^{[4]}$. This also applies to the new COVID-19 vaccines which were developed rapidly due to concerns about the high transmissibility and overall mortality rate of SARS-CoV-2. As phase I, II and III trials were combined in order to save time, it is important to identify previously unreported adverse effects in vaccine recipients. 
Iran began COVID-19 vaccination on 9 February 2021 using Sputnik V, the world's first registered COVID-19 vaccine. The Moderna, PfizerBioNTech (mRNA vaccines) ${ }^{[5,6]}$ and Sputnik V (rAd26 and rAd5 vector-based vaccine) ${ }^{[7]}$ vaccines all have over $90 \%$ efficacy. Sputnik $V$ uses a recombinant adenovirus method (adenovirus 26 and adenovirus 5) to express the SARS-COV-2 spike protein and is currently registered in more than 55 countries ${ }^{[8]}$. The efficacy of the Sputnik $\vee$ vaccine is $91.4 \%$ by 21 days after the first injection ${ }^{[9]}$ and except in one study where four cases of self-reported anaphylaxis were observed ${ }^{[10]}$, no other serious adverse reactions have been reported so far ${ }^{[8]}$. In this case report, we describe a case of severe persistent eczema in a recipient of the Sputnik $\vee$ vaccine, which developed soon after the first injection.

\section{CASE DESCRIPTION}

A 40-year-old woman, with no known illness, presented with a 2-week history of eczematous lesions on the forehead, nasal bridge, cheeks, breasts and upper limbs. The patient had received her first dose of Gam-COVID-Vac (Sputnik V) on 24 February 2021. She immediately experienced low-grade fever and mild myalgia. Fever and myalgia subsided on the second day but eczematous-like skin lesions then developed (Fig. 1). She took fexofenadine (Telfast) once daily for 2 weeks as a remedy for the skin rash. Her medical history was insignificant and she was not on any medication. She had no history of COVID-19 infection. She reported no previous allergic reactions to any medication or food. There was no family history of any allergic disease. She was non-smoker, did not drink alcohol, works as a forensics doctor in the hospital and has been exposed to COVID-19 patients. Despite consumption of a second-generation antihistamine (fexofenadine) for 2 weeks, the skin rash was sustained on most of her body except for the face (Fig. 2).

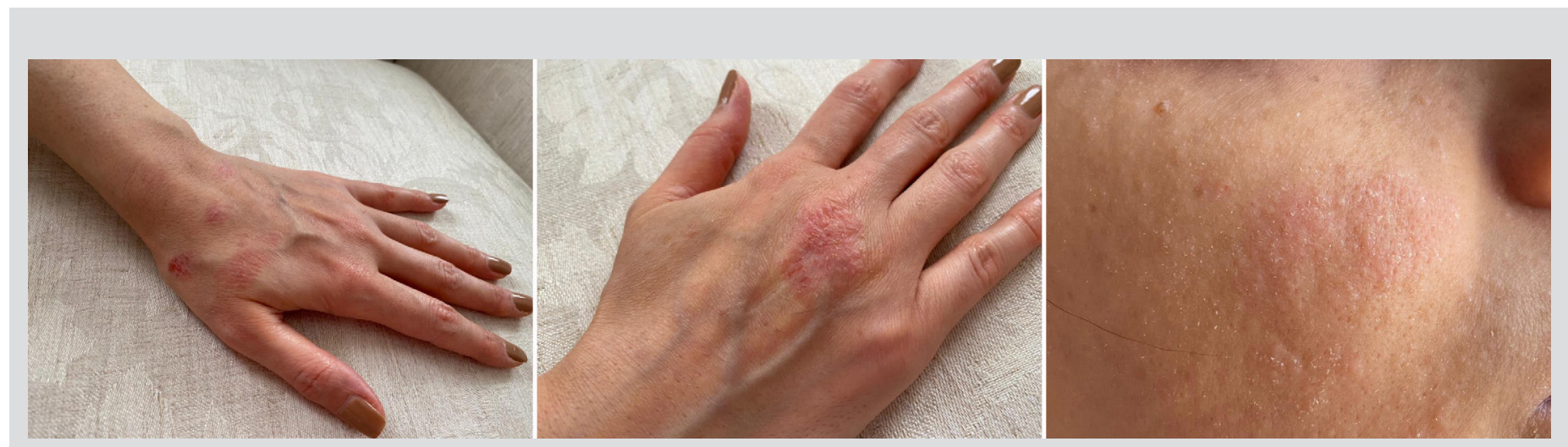

Figure 1. Severe persistent eczematous lesions on the left and right hands and on the cheek 2 days after vaccination
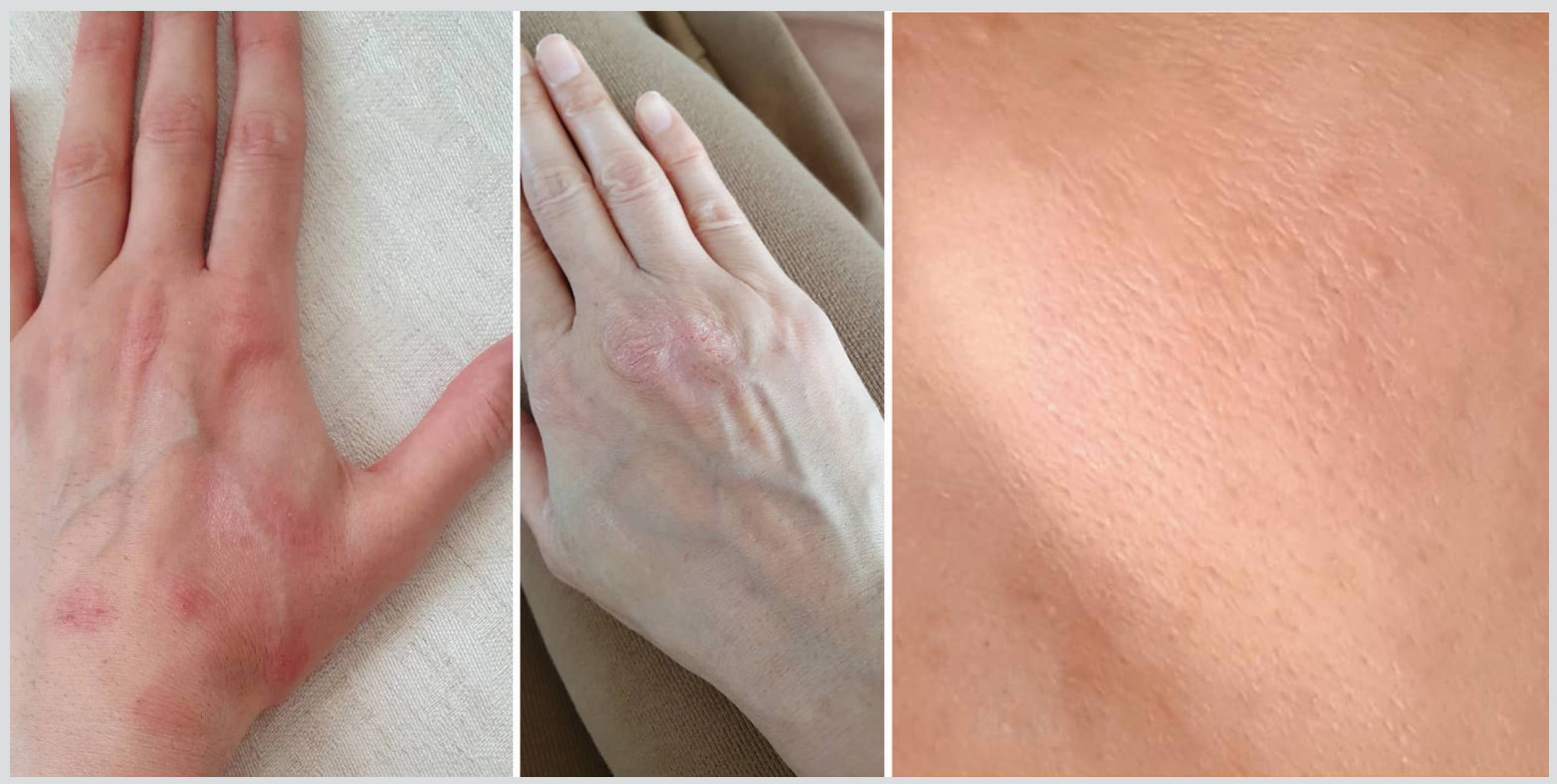

Figure 2. Partially

resolved eczematous

lesions on the left and

right hands, and on

the cheek at 1 month

follow-up 
Early laboratory results did not reveal any pathological findings. There was no leucocytosis or increases in the erythrocyte sedimentation rate (ESR), no uraemia and hepatic enzymes were not elevated. A follow-up laboratory study 1 month later revealed an elevated serum IgE level of 134 (normal range 0-87). Serological study also showed a SARS-CoV-2 IgG antibody index value of 0.2 (positive $>1.1$ ) and a SARSCoV-2 IgM antibody index value of 0.3 (positive >1.1), which indicated no immunity against COVID-19.

\section{DISCUSSION}

Negative immunity following the first dose of the Sputnik vaccine has been reported in several recent studies, with the main dermatological side effects being urticarial rashes ${ }^{[10-12]}$. We are the first to report a severe persistent eczematous reaction following injection of the first dose of the Sputnik vaccine.

Sputnik V is a COVID-19 adenoviral vector vaccine developed by the Gamaleya Research Institute of Epidemiology and Microbiology in Russia. It is a two-vector viral vaccine composed of two adenoviruses ${ }^{[13]}$. These vector viruses act as a container to carry the gene that expresses the spike protein (a surface protein which is only found in SARS-CoV-2) into human cells ${ }^{[14]}$. Once the vector virus is inside, it induces the host cell to express the spike protein and displays it on the cell surface, thus stimulating an immune response ${ }^{[14]}$. The $\mathrm{ChAdOx1}$ nCoV-19 (Oxford-AstraZeneca) vaccine employs the same mechanism of action. Given that our patient had not had any allergic issues previously, it is very likely that the vaccine was responsible for the eczematous lesions. Further studies are needed to establish the safety and efficacy of the Sputnik $\vee$ vaccine.

\section{CONCLUSION}

Rare complications of vaccines should be kept in mind during the COVID-19 pandemic. Severe persistent eczematous lesions can occur as a result of vaccination with the Sputnik V vaccine. Moreover, a severe allergic reaction to a COVID-19 vaccine may result in reduced vaccine effectiveness in the recipient.

\section{REFERENCES}

1. Our World in Data. Coronavirus (COVID-19) vaccinations. Available from: https://ourworldindata.org/covid-vaccinations?country= IRN

2. Worldometer. COVID-19 coronavirus pandemic.Available from: https://www.worldometers.info/coronavirus/.

3. Del Rio C, Collins LF, Malani P. Long-term health consequences of COVID-19. JAMA 2020;324(17):1723-1724.

4. Aragón TJ, Ulrich S, Fernyak S, Rutherford GW. Risks of serious complications and death from smallpox vaccination: a systematic review of the United States experience, 1963-1968. BMC Public Health 2003;3:26.

5. World Health Organisation. The Moderna COVID-19 (mRNA-1273) vaccine: what you need to know. Available from: https://www.who.int/news-room/feature-stories/ detail/the-moderna-covid-19-mrna-1273-vaccine-what-you-need-to-know?gclid=CjwKCAjw87SHBhBiEiwAukSeUWBMzhuDRLpoRpxUz89_6pEvA7ilA7aPwLWPNA_ ebpbKpa7NRq1HXBoCk5kQAvD_BwE.

6. World Health Organisation. Background document on mRNA vaccine BNT162b2 (Pfizer-BioNTech) against COVID-19. Available from: https://www.who.int/publications/i/ item/background-document-on-mrna-vaccine-bnt162b2-(pfizer-biontech)-against-covid-19.

7. Logunov DY, Dolzhikova IV, Shcheblyakov DV, Tukhvatulin AI, Zubkova OV, Dzharullaeva AS, et al. Safety and efficacy of an rAd26 and rAd5 vector-based heterologous primeboost COVID-19 vaccine: an interim analysis of a randomised controlled phase 3 trial in Russia. Lancet 2021;397(10275):671-681.

8. Laine C, Cotton D, Moyer DV. COVID-19 vaccine: practical clinical considerations. Ann Intern Med 2021;174(5):694.

9. CDC COVID-19 Response Team, Food and Drug Administration. Allergic reactions including anaphylaxis after receipt of the first dose of Pfizer-BioNTech COVID-19 vaccine - United States, December 14-23, 2020. MMWR Morb Mortality Wkly Rep 2021;70(2):46-51.

10. Babamahmoodi F, Saeedi M, Alizadeh-Navaei R, Hedayatizadeh-Omran A, Mousavi SA, Ovaise G, et al. Side effects and immunogenicity following administration of the Sputnik V COVID-19 vaccine in health care workers in Iran. Sci Rep 2021;11(1):21464.

11. Chahla RE, Tomas-Grau RH, Cazorla SI, Ploper D, Vera Pingitore E, López MA, et al. Long-term analysis of antibodies elicited by SPUTNIK V: a prospective cohort study in Tucumán, Argentina. Lancet Reg Health Am 2022;6:100123.

12. Tukhvatulin AI, Dolzhikova IV, Shcheblyakov DV, Zubkova OV, Dzharullaeva AS, Kovyrshina AV, et al. An open, non-randomised, phase $1 / 2$ trial on the safety, tolerability, and immunogenicity of single-dose vaccine "Sputnik Light" for prevention of coronavirus infection in healthy adults. Lancet Reg Health Eur 2021;11:100241.

13. Jones I, Roy P. Sputnik V COVID-19 vaccine candidate appears safe and effective. Lancet 2021;397(10275):642-643.

14. Kounis NG, Koniari I, de Gregorio C, Velissaris D, Petalas K, Brinia A, et al. Allergic reactions to current available COVID-19 vaccinations: pathophysiology, causality, and therapeutic considerations. Vaccines 2021;9(3):221. 